\title{
Factors affecting the utilization of antenatal care among pregnant women: A literature review.
}

\author{
Sumera Aziz Ali ${ }^{*}$, Aftab Ahmed Dero ${ }^{2}$, Savera Aziz Ali ${ }^{3}$, Gulshan Bano Ali ${ }^{4}$ \\ ${ }^{1}$ Department of Community Health Sciences, Aga Khan University, Karachi, Pakistan \\ ${ }^{2}$ The Department of Pediatrics and Child Health, Aga Khan University Hospital, Karachi, Pakistan \\ ${ }^{3}$ The Pediatrics Intensive Care Unit, Aga Khan University Hospital, Karachi, Pakistan \\ ${ }^{4}$ Centre for Epidemiology and Biostatistics, Melbourne School of Population and Global Health, Carlton, Australia
}

\begin{abstract}
Antenatal care is the care given to pregnant women in order to have a safe pregnancy and a healthy baby. Antenatal care is an important determinant of high maternal mortality rate and one of the basic components of maternal care on which the life of mothers and babies depend. Thus, Antenatal care is a key strategy to improve maternal and infant health. Different studies have found that inappropriate antenatal care has been associated with adverse pregnancy outcomes. Recently the technical working group of World Health Organization has recommended a minimum level of care to be eight visits throughout the pregnancy to reduce the maternal morbidity and mortality. Several studies conducted in different countries on demographic and socio-cultural factors influencing use of maternal health care services, have shown that factors like maternal age, number of living children, education, place of residence, occupation, religion and ethnicity are significantly associated with use of antenatal care. The findings of various factors associated with utilization of antenatal care have not been synthesized collectively. Therefore, there was a need to carry out a literature review to synthesize findings collectively regarding the factors affecting the utilization of antenatal care. Hence the objective of this literature review was to appraise the factors affecting anternatal care utilization among pregnant women. The findings of this literature review could help in planning and developing strategies for utilization of antenatal care ANC among pregnant women.
\end{abstract}

Keywords: Antenatal care, Factors, Utilization, Pregnancy.

Accepted on September 14, 2018

\section{Introduction}

\section{Antenatal care}

Antenatal care is the care given to pregnant women so that they have safe pregnancy and healthy baby [1]. The provision of antenatal care (ANC) services brings with it a positive impact on pregnancy as it enables the identification of risk factors and early diagnosis of pregnancy complications like preterm delivery and appropriate management [2]. The positive impact can be achieved through screening for pregnancy problems, assessing pregnancy risk, treating problems that may arise during the antenatal period, giving medication that may improve pregnancy outcomes, providing information to the pregnant woman, preparing physically and psychologically for childbirth and parenthood $[3,4]$.

Generally, at the first antenatal visit to a healthcare facility, a pregnant woman is issued with an antenatal care card. This card is the principal record of the pregnancy and is filled in whenever the woman goes for an ANC visit. After the first visit, the woman is considered to be booked for subsequent ANC visits to identify the complications like preterm delivery and manage these complications in timely manner [5]. The first visit is important because that is when a woman receives a complete assessment of gestational age and the risk factors [6]. A full and relevant medical history is taken from the pregnant woman including current pregnancy, previous pregnancies, previous history of preterm birth, complications and outcomes, medical problems, including psychiatric problems and previous operations, familial and genetic disorders, allergies, use of medications, use of alcohol, tobacco and other substances and family and social circumstances [6]. A physical examination is done which is divided into three categories including a general examination, which includes weight, height, heart rate, the color of mucus membranes, blood pressure, check for edema, and palpitations of lymph nodes. In addition, a systematic examination, includes examination of teeth, gums, breasts, thyroid, and heart and lung functions [6]. Finally, pregnancy related examination includes inspection and palpitation of the pregnant uterus, with measurement of the symphysis-fundal height in centimeters. After that, pregnant women undergo essential screening investigations, which include syphilis serology, rhesus (D) blood group, hemoglobin (Hb) level, human immunodeficiency virus and protein and glucose levels in urine [6]. All pregnant women are given supplements of ferrous sulphate tablets to prevent anemia, calcium tablets to prevent complications from pre-eclampsia, folic acid, and tetanus toxoid to prevent neonatal tetanus. Recently, it is the 
Citation: Ali SA, Dero AA, Ali SA, et al. Factors affecting the utilization of antenatal care among pregnant women: A literature review. J Preg

recommendation of Word Health Organization that pregnant women should have their first contact in the first 12 weeks' gestation, with subsequent contacts taking place at 20,26, 30, 34, 36, 38- and 40-weeks' gestation [7]. Hence, a minimum of eight contacts is recommended to reduce perinatal mortality and improve women's experience of care [7]. In addition, ANC along with family planning, skilled delivery care, and emergency obstetric care, is a key element of the package of services aimed at improving maternal and newborn health [8].

A number of studies have identified the lack of antenatal care as a risk factor for maternal morbidity and mortality [9-11]. Since inadequate $\mathrm{ANC}$ is associated with worse pregnancy outcomes, it is vital for health policymakers to better understand the factors influencing proper and prompt utilization of ANC. Utilization of services during pregnancy will lead to further utilization of additional maternal services like institutional delivery and seeking assistance for complications during delivery and postnatal period. Although, individual studies have highlighted many factors affecting the utilization of antenatal care in different contexts, these findings have not been synthesized collectively. Therefore, there was a need to carry out a literature review to synthesize findings regarding the factors affecting the

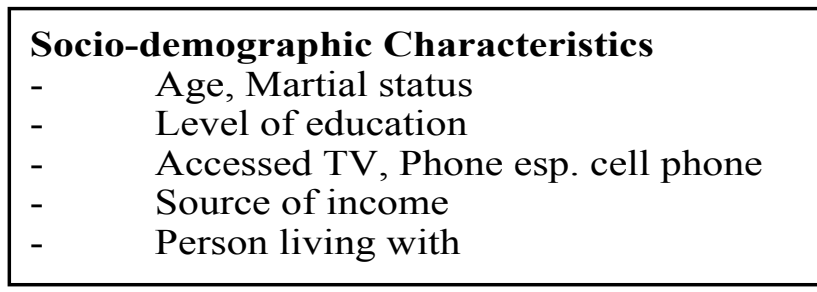

\section{Socio-cultural factors}

- Unplanned Pregnancy

Fear of testing for HIV status

Limited knowledge about ANC benefits

Peer influence

TBA influence

Low decision-making authority

\begin{tabular}{|ll}
\hline \multicolumn{2}{l}{ Obstetric factors } \\
- & Gravida \\
- & Parity \\
- & Complications during pregnancy
\end{tabular}

\begin{tabular}{|ll|}
\hline \multicolumn{2}{|c|}{ Economical factors } \\
- & Financial constraints (Poverty) \\
- & High ANC fees \\
\hline
\end{tabular}

$$
\begin{array}{ll}
\text { Facility factors } \\
- & \text { Distance to the health centre } \\
- & \text { Delay in attending to clients } \\
- & \text { Quality of care }
\end{array}
$$

utilization of ANC. Hence the objective of this literature review was to appraise the factors affecting ANC utilization among pregnant women. The findings of this study could help in planning and developing strategies for the utilization of ANC among pregnant women.

\section{Factors associated with utilization of antenatal care}

The ability to utilize ANC services in developing countries is affected by a number of factors [12,13]. According to Andersen and Newman's health behavioral model [14] (Figure 1), individual determinants of health care utilization can be divided into predisposing, enabling and need components $[15,16]$. This model helped us to conceptualize the factors associated with ANC utilization and was also used to do the focused literature search in order to find out the factors associated with antenatal care utilization for this review.

With respect to $\mathrm{ANC}$, predisposing determinants refer to individual characteristics which exist prior to the pregnancy and affect the propensity to use care. Previous studies have concluded that young age, low educational level, lack of a paid job, poor language proficiency, support from a social network and lack of knowledge of the health care system are associated

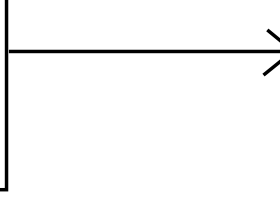


with inadequate ANC utilization [16,17]. Enabling determinants refer to conditions which make ANC available to pregnant women. The absence of health insurance, the planned pattern of ANC, hospital type at booking, personalized communication, and knowledge of cultural practices of the care provider have been found to be associated with inadequate ANC services utilization $[15,16]$. The pregnancy-need components of the determinants include pregnancy-related elements explaining the degree of care needed [16,17]. Inadequate use of ANC seems to be related to high parity, unplanned pregnancy, no previous premature birth, discontinuity of care, late recognition of pregnancy and behavioral factors such as smoking during pregnancy $[16,17]$. Some of the important factors are discussed below in greater detail.

\section{Maternal age}

Results from various studies have found mixed evidence of an association between age and utilization of ANC services. In some studies, young age of women has been identified as a predisposing determinant for utilization of ANC services [18]. However, few studies suggest contrary to these studies, few studies suggest that increased age is associated with more utilization of ANC services [19,20]. For example, study from Central Ethiopia found that the odds of attending ANC are 1.2 times higher $(\mathrm{OR}=1.168)$ for women in the age group of 20-34 as compared to those in the age group 15-19 women [21]. Similarly, a study conducted in Vietnam found that older women (more than 25 years old) were more likely to utilize ANC [22]. Likewise, a study conducted in China also found that women between the ages of 25 and 30 and women older than 30 were more likely to have adequately utilized antenatal care $(\mathrm{AOR}=2.2$ and $1.9,95 \% \mathrm{CI}=1.4-3.5$ and 1.1-3.2, respectively) than younger women [23].

\section{Women's education}

Educated women tend to have a greater awareness of the existence of ANC services and the advantages of using such services [20]. It is argued that educated women were more aware of health problems, know more about the availability of health care services, and utilize the information more effectively than non-educated women [24]. Moreover, higher levels of education tend to positively affect health-seeking behaviors, and education may increase a woman's control over her pregnancy [25]. In addition, education may help to expose women to more health education messages and campaigns, enabling them to recognize danger signs and complications and take appropriate action [25]. These women might have greater opportunities to receive health information and pay more attention to maternal healthcare [25]. Studies have shown that women with lower education usually have less knowledge about ANC services and more difficulties to get access to ANC services [22]. A study conducted in Central Ethiopia found that women with some education were more than two times more likely to attend $\mathrm{ANC}(\mathrm{OR}=2.645)$ as compared with those who had no education [21] and similar findings were found in the study conducted in North Ethiopia, Nigeria and China [24-26].

\section{Socio-economic status}

Financial difficulties have been considered as an important barrier to antenatal care for migrant women [25]. Most of the studies have shown a positive association between socioeconomic status and the utilization of ANC [20]. A study from Ethiopia identified that when women with higher incomes tend to start ANC early and the likelihood of utilizing ANC decreased, as the family income gets lower [21]. Likewise, a study from China found that women who had higher household income were more likely to have adequately utilized ANC services $\quad(A O R=1.6,95 \% \quad C I=1.0-2.5)[25]$. The positive contribution of better wealth status for all maternity service indicators and its significant contribution to postnatal care are also observed in other studies [26].

\section{Parity}

Studies have suggested that parity influences initiation of ANC, as parity increases, the experience of timely initiation of ANC decreases [22]. High parity women might tend to rely on their experiences from previous pregnancies and not feel the need for antenatal care [25]. Due to their greater level of experience, these women might feel more confident during pregnancy and consider antenatal care to be less important [25]. This was evidenced by findings in different studies in which respondents with first pregnancy were about two times more likely to book early than those with more children [27].

\section{History of reproductive loss and previous pregnancy complications}

A history of reproductive loss has proved to be a strong predictor of early ANC initiation [27]. The researchers determined that women who had previously experienced miscarriages or stillbirths are more likely to utilize ANC services as compared to their counterparts [25].

\section{Supportive spouse or partner}

Having a spouse or partner who is not supportive was reported to be associated with initiating ANC late for both adolescents and adult women $(p=0.035)$ [27]. In of the studies, the researchers concluded that women who had no support from their spouses or partners utilized ANC services almost three weeks later than those who were given support [27]. Similarly, the utilization of ANC was almost nine times more likely for women reported their husbands to approve ANC than women with those whose husbands did not approve ANC service $(\mathrm{OR}=8.99)[28]$.

\section{Women's knowledge of antenatal care}

Health knowledge is an important factor. It enables women to be aware of their rights and health status in order to seek appropriate health services [24]. The odds of utilizing ANC were more than three times for those with better knowledge of danger signs of pregnancy than those with poor knowledge $(\mathrm{OR}=3.541)$ [21]. The studies have revealed that sufficient knowledge of the benefits of ANC and of the complications associated with pregnancy plays an important role in the utilization of ANC services. In of the studies conducted by 
Citation: Ali SA, Dero AA, Ali SA, et al. Factors affecting the utilization of antenatal care among pregnant women: A literature review. J Preg

Rosliza and Muhamad, no significant relationship ( $\mathrm{p}=0.279)$ was found between knowledge of ANC and early antenatal booking [29]. They discovered that pregnant women's level of knowledge of the importance of ANC, screening tests, and complications of diabetes and hypertension during pregnancy was poor [28].

\section{Quality of care}

Women were reported to initiate ANC late owing to the perceived bad quality of service at the healthcare facility [27]. The women's criticisms were related mainly to lack of services, citing reasons such as being sent home without receiving services owing to insufficient staff, and having to purchase drugs, cards or diagnostic tests, although the service was supposed to be free [27]. Another strong facility level predictor for skilled maternal care utilization was the performance of health facilities. The presence of all the six signal functions in the nearby basic essential obstetric care facility (health center) positively contributes to the utilization of all indicators of skilled maternal services. Functioning obstetric facility means performing the essential services for normal situations and complications and these services should be available 24 hours a day and 7 days a week. The presence of all signal functions reflects better performance (quality) of a health facility [26].

\section{Distance to a healthcare facility}

The research has also revealed a very strong association between distance and attendance of ANC. Generally, the distance has been identified as an important barrier to the use of services, especially in rural areas [29]. Studies have revealed that general health care utilization for every kind of service is affected by distance from those services. There was a decay effect of the distance on the health care service utilization, i.e., as the distance increases from the healthcare facilities; utilization of services was reduced [30-32]. Generally, the effect of distance on the use of services increases when it is combined with lack of transportation particularly in developing countries [29]. Moreover, access to the facilities also has an effect on the frequencies of services being used [29]. Studies from Pakistan have found that access to obstetric care depends upon the transportation system and physical distance between the villages and the centers [33]. Moreover, with huge expenditures and passage of twenty-two years, only $33 \%$ of the rural Pakistani population is living within access of 5 kilometers $(\mathrm{km})$ [34]. This distance has even been found as a hindrance in seeking care especially in the case of women who lacks autonomy and needs somebody to accompany her [35]. As a result, the factor of distance gets strongly adhered to other factors such as the availability of transport, the total cost of travel and women's restricted mobility [35].

Likewise, other studies have also found that an increase in distance to the nearest health facility led to fewer antenatal visits [36]. A strong association between distance to the health facility and utilization of ANC services was reported by another study [24]. Thus, distance to the antenatal clinics has proved to be a problem that tends to limit access to the antenatal service [37]. In trying to explain the association, the researchers argue that many pregnant women find it distressing to walk long distances or take two or more taxis to a health facility; therefore, they tend to utilize ANC services less regularly than those who live close by [24].

\section{Conclusion}

This literature review identifies multiple sociodemographic, reproductive and access related factors which affect the utilization of antenatal care among pregnant women in different countries. Several studies conducted in different countries have shown that factors like maternal age, number of living children, education, socioeconomic status, previous bad obstetrical history, support from spouse, quality of care and distance from health care facility are significantly associated with use of antenatal care. The findings of this literature review could help policy makers and researcheres to design some country specific strategies to improve the utilization of ANC.

\section{Competing Interest}

We declare that there is no competing interest.

\section{Availability of Data and Materials}

This was a literature review, and all cites references are available online.

\section{References}

1. Abosse Z, Woldie M, Ololo S. Factors influencing antenatal care service utilization in Hadiya zone. Ethiop J Health Sci. 2010;20.

2. Perumal N, Cole DC, Ouédraogo HZ, et al. Health and nutrition knowledge, attitudes and practices of pregnant women attending and not-attending ANC clinics in Western Kenya: a cross-sectional analysis. BMC Preg Childbirth. 2013;13:1.

3. Kisuule I, Kaye DK, Najjuka F, et al. Timing and reasons for coming late for the first antenatal care visit by pregnant women at Mulago hospital, Kampala Uganda. BMC Preg Childbirth. 2013;13:1.

4. WHO Global Health Observatory (GHO): Antenatal caresituations and trends. 2011.

5. Finlayson K, Downe S. Why do women not use antenatal services in low-and-middle-income countries? A metasynthesis of qualitative studies. PLoS Med. 2013; 10:e1001373.

6. Women's NCC, Health Cs. Antenatal care. 2008.

7. Organization WH. New guidelines on antenatal care for a positive pregnancy experience. Sexual Reprod Health. 2016.

8. Pell C, Menaca A, Were F, et al. Factors affecting antenatal care attendance: results from qualitative studies in Ghana, Kenya, and Malawi. PloS one. 2013;8:e53747.

9. Bauserman M, Lokangaka A, Thorsten V, et al. Risk factors for maternal death and trends in maternal mortality in lowand middle-income countries: a prospective longitudinal cohort analysis. Reprod Health. 2015;12(2):S5. 
10. Carroli G, Rooney C, Villar J. How effective is antenatal care in preventing maternal mortality and serious morbidity? An overview of the evidence. Paediatr Perinat Epidemiol. 2001;15:1-42.

11. McDonagh M. Is antenatal care effective in reducing maternal morbidity and mortality? Health Policy and Planning. 1996;11:1-15.

12. Gupta S, Yamada G, Mpembeni R, et al. Factors associated with four or more antenatal care visits and its decline among pregnant women in Tanzania between 1999 and 2010. PloS One. 2014;9:e101893.

13. Farah S, Karim M. Determinants of utilization of antenatal care services in rural area of Bangladesh. Bangladesh Med J. 2016;44:67-71.

14. Boerleider AW, Wiegers TA, Mannien J, et al. Factors affecting the use of prenatal care by non-western women in industrialized western countries: a systematic review. BMC Preg Childbirth. 2013;13:1.

15. Beeckman K, Louckx F, Putman K. Content and timing of antenatal care: predisposing, enabling and pregnancyrelated determinants of antenatal care trajectories. Eur J Public Health. 2013;23:67-73.

16. Feijen-de Jong EI, Jansen DE, Baarveld F, et al. Determinants of late and/or inadequate use of prenatal healthcare in high-income countries: a systematic review. Eur J Public Health. 2012:164.

17. Heaman M, Bayrampour H, Kingston D, et al. Migrant women's utilization of prenatal care: a systematic review. Matern Child Health J. 2013;17:816-36.

18. Nketiah-Amponsah E, Senadza B, Arthur E. Determinants of utilization of antenatal care services in developing countries: recent evidence from Ghana. African J Econ Manag Stud. 2013;4:58-73.

19. Roy MP, Mohan U, Singh SK, et al. Determinants of utilization of antenatal care services in rural Lucknow, India. J Family Med Prim Care. 2013;2:55.

20. Efendi F, Chen CM, Kurniati A, et al. Determinants of utilization of antenatal care services among adolescent girls and young women in Indonesia. J Women Health. 2016:1-16

21. Birmeta K, Dibaba Y, Woldeyohannes D. Determinants of maternal health care utilization in Holeta town, central Ethiopia. BMC Health Serv Res. 2013;13:1.

22. Tran TK, Gottvall K, Nguyen HD, et al. Factors associated with antenatal care adequacy in rural and urban contextsresults from two health and demographic surveillance sites in Vietnam. BMC Health Serv Res. 2012;12:1.

23. Zhao Q, Huang ZJ, Yang S, et al. The utilization of antenatal care among rural-to-urban migrant women in Shanghai: a hospital-based cross-sectional study. BMC Public Health. 2012;12:1.

24. Onasoga OA, Afolayan JA, Oladimeij BD. Factors influencing utilization of antenatal care services among pregnant women in Ife Central Lga, Osun State Nigeria. Adv Appl Sci Res. 2012;3:1309-15.

25. Zhao QZJH, Yang S, Pan J, et al. The utilization of antenatal care among rural-to-urban migrant women in Shanghai:a hospital-based cross-sectional study. BMC Public Health. 2012;12.

26. Worku AG, Yalew AW, Afework MF. Factors affecting utilization of skilled maternal care in Northwest Ethiopia: a multilevel analysis. BMC Int Health Hum Rights 2013;13:1.

27. Gross K, Alba S, Glass TR, et al. Timing of antenatal care for adolescent and adult pregnant women in south-eastern Tanzania. BMC Preg Childbirth. 2012;12:1.

28. Rosliza A, Muhamad J. Knowledge, attitude and practice on antenatal care among orang asli women in Jempol, Negeri Sembilan. Malaysian J Public Health Med. 2011;11:13-21.

29. Ali NR, Luby S, Hossein Rahbar M. Does use of a government service depend on distance from the health facility? Health Policy and Planning. 1999;14:191.

30. Yamashita T, Kunkel SR. The association between heart disease mortality and geographic access to hospitals: County level comparisons in Ohio, USA. Social Science \& Medicine. 2010;70:1211-8.

31. Arcury TA, Gesler WM, Preisser JS, et al. The effects of geography and spatial behavior on health care utilization among the residents of a rural region. Health Serv Res. 2005;40:135-55

32. Lin G. A GIS Method to Assess Distance Effects on Hospitalizations. Unpublished research paper, Department of Geology and Geography, West Virginia University. 2002.

33. Midhet F, Becker S, Berendes HW. Contextual determinants of maternal mortality in rural Pakistan. Soc Sci Med. 1998;46:1587-98.

34. World Health Organisation Health Systems Profile-Pakistan EMRO Regional Health Systems Observatory, Cairo. 2007.

35. Shaikh BT, Hatcher J. Health seeking behavior and health service utilization in Pakistan: challenging the policy makers. J Public Health. 2005;27:49-54.

36. Nicholas NA, Kyei OMRC, Sabine G. The Influence of Distance and Level of Service Provision on Antenatal Care Use in Rural Zambia. PLoS One. 2012.

37. Openshaw MR, Bomela HN, Pretlove S. An evaluation of the timing and use of healthcare during pregnancy in Birmingham, UK and Pretoria, South Africa. ISRN Obstet Gynecol. 2011.

\section{*Correspondence to}

Sumera Aziz Ali

Department of Community Health Sciences

Aga Khan University

Pakistan

E-mail: sumera.ali@aku.edu 